\title{
Origin of Strong Coupling in Lithium under Pressure
}

\author{
Deepa Kasinathan, ${ }^{\text {a }}$ K. Koepernik, ${ }^{\text {b }}$ J. Kuneš, ${ }^{\text {a,c }}$ H. Rosner, ${ }^{\text {d W. E. Pickett }}{ }^{\text {a,* }}$ \\ a Department of Physics, University of California Davis, Davis, CA 95616 \\ ${ }^{\mathrm{b}}$ IFW Dresden, P. O. Box 270116, D-01171 Dresden, Germany \\ ${ }^{\mathrm{c}}$ Institute of Physics, ASCR, Cukrovarnická 10, 16253 Praha 6, Czech Republic \\ ${ }^{\mathrm{d}}$ Max-Planck-Institut für Chemische Physik fester Stoffe Dresden, Germany
}

\begin{abstract}
In an attempt to provide a clearer understanding of the impressive increase in $\mathrm{T}_{c}$ under pressure in elemental Li, linear response calculation of the phonon dispersion curves, electron-phonon matrix elements, phonon linewidths and mode $\lambda$ 's have been carried out on a finer mesh $\left(24^{3}\right.$ in the Brillouin zone) than done previously, for the volume corresponding to 20 GPa pressure. The result illustrates the great need for a fine mesh (even finer than this) for converged results of $\lambda$ and the spectral function $\alpha^{2} F$. Although the initial pressure-induced transverse $\mathcal{T}_{1}$ phonon instability (in harmonic approximation) near the symmetry point $\mathrm{K}$ has dominated attention, the current results show that the high value of $\mathrm{T}_{c}$ gets strong contributions from elsewhere in the zone, particularly from the longitudinal mode along (100).
\end{abstract}

Key words: Superconductivity, Lithium, High pressure, Strong coupling.

$P A C S:$...

\section{Introduction}

Elemental lithium, with superconducting $\mathrm{T}_{c} \sim 15 \mathrm{~K}$ around[1,2,3] $30 \mathrm{GPa}$ and up to $20 \mathrm{~K}$ in strained samples[1] at $50 \mathrm{GPa}$, joins $\mathrm{MgB}_{2}\left(\mathrm{~T}_{c}=40 \mathrm{~K}\right)$, metallic $\mathrm{Y}\left(\mathrm{T}_{c}=20\right.$ $\mathrm{K})[4]$, and $\mathrm{PuCoGa}_{5}\left(\mathrm{~T}_{c}=19 \mathrm{~K}\right)[5]$ as the big surprises in critical temperature in this century. Transformation from a simple nearly-free-electron metal not superconducting[6] above $100 \mu \mathrm{K}$ to a strongly coupled electron-phonon superconductor at 20-50 GPa has been explained, at least semiquantitatively, in recent work by Profeta et al.[7] and by Kasinathan et al. [8]. The evolution of $\mathrm{T}_{c}$ has been linked to the increase in electron-phonon coupling of specific phonon branches along intersections of Kohn anomaly surfaces with pressure. The coupling strength is quite strongly dependent on the phonon wave vector $Q$ and phonon polarization. In particular, the $<1 \overline{1} 0>$ polarized phonon along the (110) $\Gamma-\mathrm{K}$ direction softens with increasing pressure and finally becomes unstable.

The nesting function $\xi(Q)$ which describes the phase space for the electron-hole scattering processes across the Fermi surface has fine structure[8] requiring a very dense mesh to obtain accurate values where its amplitude is

\footnotetext{
* Corresponding author.

Email address: pickett@physics.ucdavis.edu (W. E. Pickett).
}

large. Since the electron-phonon (EP) coupling involves these same scattering events weighted by EP matrix elements that normally vary smoothly with $\mathrm{Q}$, the fine structure in $\xi(Q)$ implies corresponding fine structure in EP coupling (as contained for example in the EP contribution to the phonon linewidths $\left.\gamma_{Q \nu}\right)$. Such fine structure makes the sampling of the Brillouin zone an issue if accurate numerical results for $\alpha^{2} F$ are desired. Our previous linear response phonon calculations [8] were performed on $12^{3}$ mesh (72 $Q$ points in the irreducible zone). In the present work, we focus on the $20 \mathrm{GPa}$ case (fcc lattice constant $\mathrm{a}_{0}=6.80 \mathrm{bohr}$ ) where strong phonon softening arises but structural instability has not yet occurred. The phonon energies and the Eliashberg spectral function constants have been presently calculated on a much finer $24^{3}$ mesh $(413 Q$ points in the irreducible zone). Careful examination of the results lead to a revised picture of what coupling is important for $\mathrm{T}_{c}$ (as opposed to what is important in increasing $\lambda$, which is a far simpler question).

\section{Computational Details}

The electronic wave functions are calculated using a full potential linear muffin tin orbital (FP LMTO) method as 


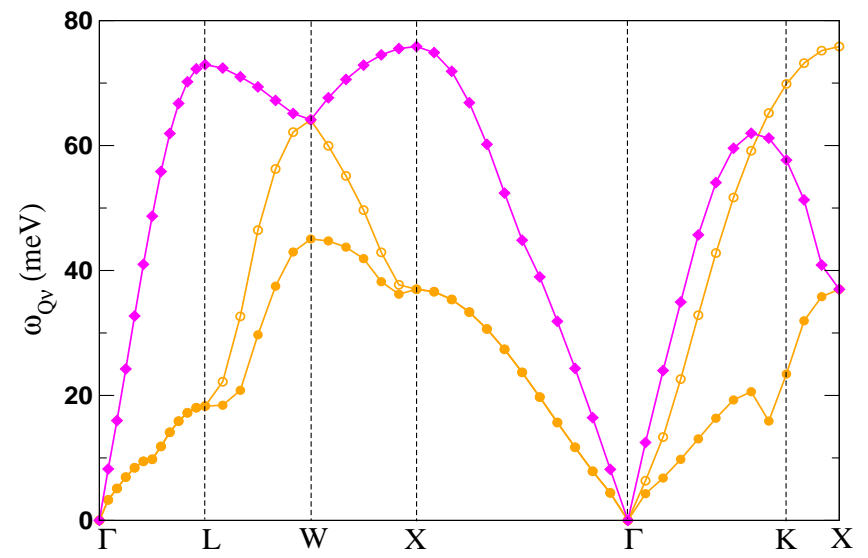

Fig. 1. (color online) Calculated phonon spectrum for fcc Li at 20 GPa along high symmetry directions. The solid diamonds denote the longitudinal mode $\mathcal{L}$, and where the transverse branches are non-degenerate, solid circles denote the transverse mode $\mathcal{T}_{1}$ and open circles denote the transverse mode $\mathcal{T}_{2}$. The mode symmetry has been used to connect branches across crossings $(\Gamma-\mathrm{K})$ although this is not possible off symmetry lines where branches do not cross.

implemented by Savrasov [10]. We have used the VoskoWilk-Nusair local exchange-correlation [11]. The phonon energies and the coupling constants have been calculated using linear response theory [10]. Phonons were calculated for a dense $24^{3}$ mesh corresponding to 413 inequivalent $Q$ points in the irreducible zone. The electronic BZ zone integration was performed with a $40^{3} \mathrm{k}$ point grid.

\section{Dispersion Throughout the Zone}

The calculated phonon dispersion curves are displayed in Fig. 1. The only noticeable softening is seen midway along the $\Gamma$-L direction (transverse $\mathcal{T}$ branches), for the $<$ $1 \overline{1} 0>$ polarized $\mathcal{T}_{1}$ branch along the $\Gamma-\mathrm{K}$ direction emphasized previously, [8] and possibly softening in the $\mathcal{T}$ branches around the X point. We had identified previously that the $Q=\left(\frac{2}{3}, \frac{2}{3}, 0\right) \frac{2 \pi}{a} \mathcal{T}_{1}$ phonon (near $\mathrm{K}$ ) causes large band shifts with atomic displacement $\left(\delta \varepsilon_{k} / \delta u_{Q} \approx 5 \mathrm{eV} / \AA\right)$ near the FS necks, reflecting strong EP coupling.

The corresponding 'mode $\lambda$ ' values $\lambda_{Q, \nu}$ are presented in Fig. 2. The $\lambda_{Q, \nu}$ values for the $\mathcal{T}_{1}$ branch near $\mathrm{K}$, and also near the midpoint of the $\Gamma$-L line are larger compared to other areas in the BZ, as expected from the phonon kinks. In addition, we notice that the $\lambda_{Q, \nu}$ values for all the three branches are large all along $\Gamma$-X; this coupling is not immediately obvious from the phonon dispersion in Fig. 1 because it depends only weakly on Q.

In Fig. 3, we display the corresponding linewidths $\gamma_{Q \nu}$, which are given by $[9]$

$\gamma_{Q \nu}=2 \pi \omega_{Q \nu} \sum_{k}\left|M_{k, k+Q}^{[\nu]}\right|^{2} \delta\left(\varepsilon_{k}\right) \delta\left(\varepsilon_{k+Q}\right)$.

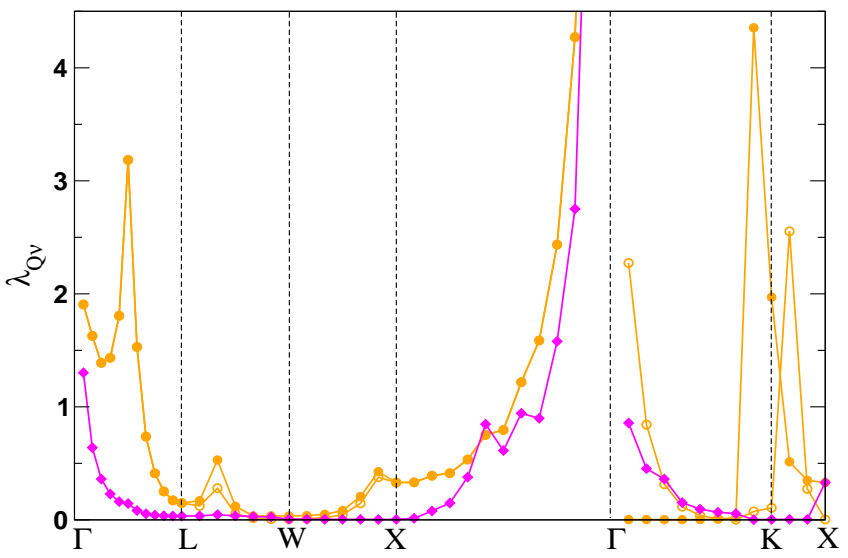

Fig. 2. (color online) Calculated mode $\lambda_{Q \nu}$ values, following the notation of Fig. 1. The landscape is dominated by peaks in the transverse branches near $\mathrm{K}$, a longitudinal peak along $\Gamma-\mathrm{L}$, and an increase around $\mathrm{Q} \sim$ zero where phase space is limited. Here $\lambda_{Q \nu}$ is normalized such that the total $\lambda$ requires a sum over the three branches (rather than an average).

While $\gamma_{Q \nu}$ includes the same Q-specific Fermi surface average of the EP matrix elements with respect to the available phase space for scattering through wavevector $\mathrm{Q}$ as does $\lambda_{Q \nu}$, it is proportional to $\omega_{Q \nu}$ rather than inversely proportional. As a result, $\gamma_{Q \nu}$ is a much better indicator of the importance of the coupling for $\mathrm{T}_{c}$. Coupling at high frequency is much more important than at low frequency for $\mathrm{T}_{c}$, whereas the opposite is true for $\lambda$. Thus it is not surprising that the linewidth 'dispersion' in Fig. 3 provides a different viewpoint than does the $\lambda_{Q \nu}$ dispersion. There is still the large contribution from the region around $\mathrm{K}$ where there is an incipient instability, but it is very limited in Q and very sharply structured, not being resolved even by the $\mathrm{Q}$ mesh we have used. The really impressive region however is the $\mathcal{L}$ branch along (100) directions, which dominates the landscape (at least along symmetry lines). There are also important contributions from the $\mathcal{L}$ branch along the $\Gamma-\mathrm{K}$ lines and from the $\mathcal{T}$ branches along (100). Even the small peak in the $\mathcal{T}$ branches midway between $\Gamma$ and L (where the kink in the $\mathcal{T}$ branch occurs) is small compared to the contribution from the $\mathcal{L}$ branch along the same line. Tse et al. present $\gamma_{Q}$ at $\mathrm{V} / \mathrm{V}_{0}=0.45 \mathrm{GPa}(\approx 50 \mathrm{GPa})$ which have some features [12] in common with Fig. 3, but cannot be compared in detail because of the different volumes.

\section{Spectral Functions and $\mathbf{T}_{c}$}

The phonon frequency distribution $F(\omega)$ is shown in Fig. 4 where it is clear that the coarser Q mesh had given a good picture of the density of states. This insensitivity does not persist when coupling is included. The Eliashberg spectral function $\alpha^{2} F(\omega)$ from both meshes is plotted in Fig. 5. For this $20 \mathrm{GPa}$ case a large broad peak dominates the 15-35 $\mathrm{meV}$ range, with less intense coupling extending on up to 


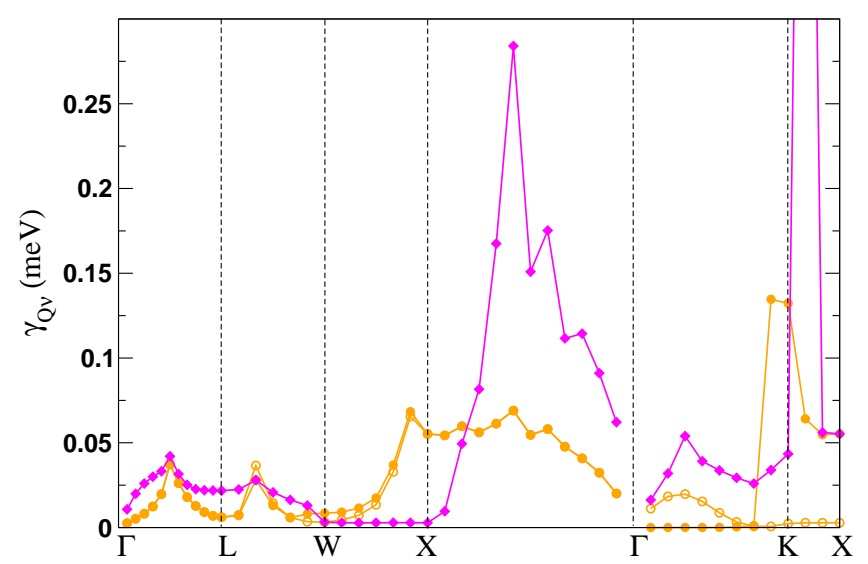

Fig. 3. (color online) Calculated linewidths $\gamma_{Q \nu}$, following the notation of Fig. 1. The landscape here is dominated by all modes along $\Gamma-\mathrm{X}$, and the region near $\mathrm{K}$.

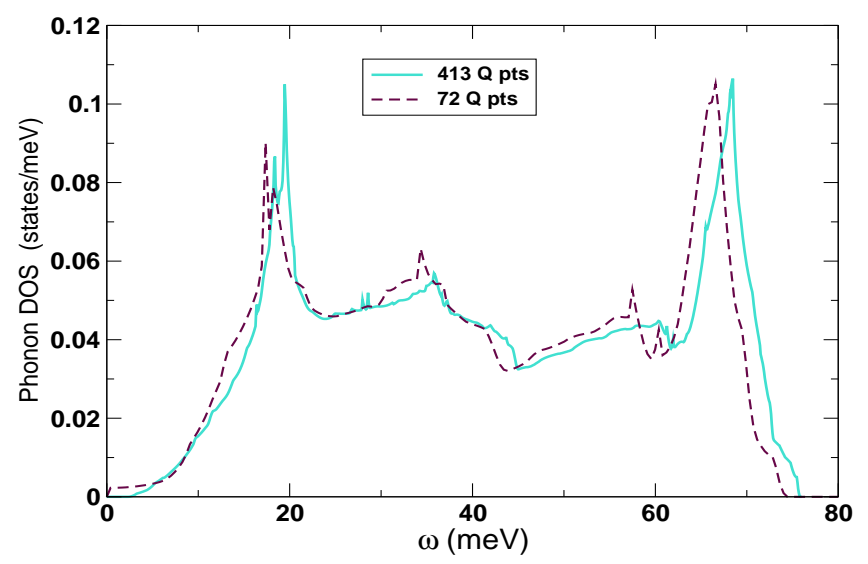

Fig. 4. (color online) Phonon density of states calculated from the $12^{3} \mathrm{Q}$ grid (72 points) and the $24^{3}$ grid (413 points), illustrating that the representation of the phonon spectrum changes little. The substantial changes in $\alpha^{2}(\omega)$ shown in Fig. 5 are discussed in the text.

$70 \mathrm{meV}$. Compared to its value from the coarser mesh, it is much smaller in the 7-20 meV range. The intensity in the low frequency region is clearly from the transverse branch phonons, while the less impressive (but still substantial) intensity in the high frequency region is from the longitudinal phonon branch.

The frequency resolved coupling strength $\alpha^{2}(\omega)$, also shown in Fig. 5 is dramatically changed by using the finer $\mathrm{Q}$ mesh. There is now a broad peak around $12 \mathrm{meV}$, still indicative of comparatively stronger coupling in the low frequency regime. However, the overriding peak in the 7-16 $\mathrm{meV}$ range has disappeared, showing that is was an artifact of the sampling of the volume near the point K using only a $12^{3}$ mesh. This feature is reminiscent of results calculated by Yin et al., [14] who have found an even narrower peak in $\alpha^{2}(\omega)$ growing at lower frequencies $(2-5 \mathrm{meV})$ in elemental $\mathrm{Y}$ under pressure. This peak also was traced to incipient instability for a transverse branch near the zone boundary.

We now concentrate on the difference in $\mathrm{T}_{c}$, and the in-

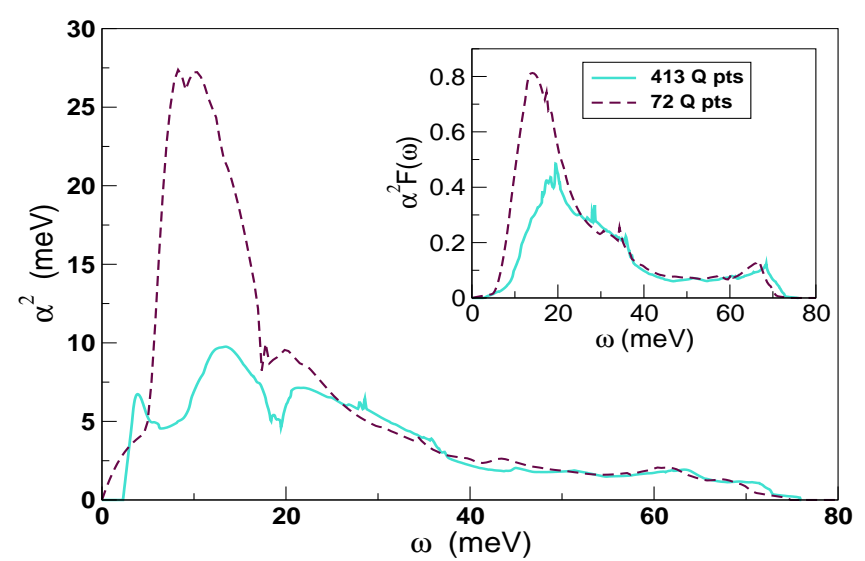

Fig. 5. (color online) Comparison of the Eliashberg spectral function $\alpha^{2} F(\omega)$ (inset) and the frequency-resolved coupling strength $\alpha^{2}(\omega)$ for fcc $\mathrm{Li}$ at $20 \mathrm{GPa}$, for both $\mathrm{Q}$ point meshes.

Table 1

From the calculated $\alpha^{2} F(\omega)$ at $20 \mathrm{GPa}$ for both $Q$ meshes, this table provides: the logarithmic, first, and second moments of the frequency; the value of $\lambda ; \mathrm{T}_{c}(\mathrm{~K})$ calculated using $\mu^{*}=0.13$ and $\mu^{*}=0.20$; and two simple measures of $\alpha^{2} F$ (see text).

\begin{tabular}{|c|c|c|c|c|c|c|c|c|}
\hline $\begin{array}{c}Q \\
\text { mesh }\end{array}$ & $\begin{array}{r}\omega_{l o g} \\
(\mathrm{~K})\end{array}$ & $\omega_{1}$ & $\omega_{2}$ & $\lambda$ & $\mathrm{T}_{c}$ & $\mathrm{~T}_{c}$ & $\lambda \omega_{1}$ & $\sqrt{\lambda \omega_{2}^{2}}$ \\
$(\mathrm{~K})$ & & $\left(\mu^{*}=0.13\right)$ & $\left(\mu^{*}=0.20\right)$ & $(\mathrm{K})$ & $(\mathrm{K})$ \\
\hline \hline $12^{3}$ & 81 & 115 & 176 & 3.1 & 20 & 16 & 357 & 310 \\
$24^{3}$ & 217 & 255 & 297 & 1.0 & 13 & 3.4 & 250 & 294 \\
\hline
\end{tabular}

terpretation of the distribution of the coupling, that we obtain from our denser $\mathrm{Q}$ mesh of data (we note that the quantities we discuss are still not fully converged). The results of this much better zone sampling are displayed in Table 1. Startlingly, the value of $\lambda$ has dropped from 3.1 to 1.0, (which was also obtained by Profeta et al. [7] )reflecting the fact that the low energy region of $\alpha^{2} F$ was (and still is) reliant on large contributions (from near $\mathrm{K}$ and perhaps elsewhere) that vary sharply with Q. For the determination of $\mathrm{T}_{c}$, the strong reduction in $\lambda$ is considerably compensated by increases in the frequency moments. The logarithmic moment $\omega_{\text {log }}$ increases by a factor of 2.7 , and the second moment by $40 \%$. At a similar volume, Shi and Papaconstantopoulos [13] obtain $\lambda=0.5$ from rigid muffin tin calculations. One source of difference is the value $\langle\omega\rangle \approx$ $325 \mathrm{~K}$ (scaled from the $\mathrm{P}=0$ Debye frequency which is our calculated value of $\omega_{1}$.

We have obtained $\mathrm{T}_{c}$ using the Allen-Dynes equation[15]

$T_{c}=\frac{\omega_{\log }}{1.2} \Lambda_{1} \Lambda_{2} \exp \left\{-\frac{1.04(1+\lambda)}{\lambda-\mu^{*}(1+0.62 \lambda)}\right\}$

where $\Lambda_{1}, \Lambda_{2}$ are strong coupling corrections that depend on $\lambda, \mu^{*}$, and the ratio $\omega_{2} / \omega_{\log }$, which is a measure of the shape of $\alpha^{2} F$. These $\Lambda$ factors are important for $\lambda=3.1$ but have little effect for $\lambda \sim 1$ or less. For the commonly used value of $\mu^{*}=0.13$, our more nearly converged results give $\mathrm{T}_{c}=13 \mathrm{~K}$ vs. the earlier estimate of $20 \mathrm{~K}$, only a $35 \%$ drop even though $\lambda$ decreases by more than a factor of three. For $\mu^{*}=0.20, \mathrm{~T}_{c}=3.4 \mathrm{~K}$. These values should be compared 


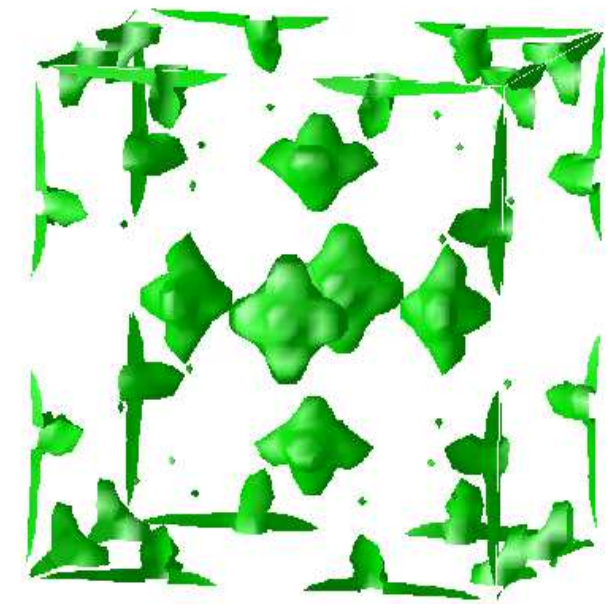

Fig. 6. (color online) Isosurface plot of $\gamma_{Q \nu}=0.054 \mathrm{meV}$ for the longitudinal branch. The box contains the $\Gamma$ point at the center and at each corner for this fcc structure. The amplitude is high inside the jack-shaped region midway between $\Gamma$ and $\mathrm{X}$, corresponding to the large linewidths evident in Fig. 3. [Unfaithful interpolation at the edges of the box account for the extra structure in those regions; the box edges are also $\Gamma-\mathrm{X}-\Gamma$ lines.]

with the nearly hydrostatic value $\mathrm{T}_{c}=6 \mathrm{~K}$ at $20 \mathrm{GPa}$ found by Deemyad and Schilling.[2]

\section{Analysis and Summary}

This apparent weak dependence of $\mathrm{T}_{c}$ on $\lambda$ reflects the observation, made several times in the past,[16] that, for providing an estimate of $\mathrm{T}_{c}$ with averages of $\alpha^{2} F$, the combination of $\lambda$ and some frequency moment separately is not the best choice. This observation is connected with the observation that coupling at low frequency, which strongly enhances $\lambda$, has only a weak effect on $\mathrm{T}_{c}$. Carbotte and collaborators [16] argued that the product $\lambda \omega_{1}$ (with $\omega_{1}$ being the first moment as defined by Allen and Dynes[15]) is nearly proportional to $\mathrm{T}_{c}$ for $1.2 \leq \lambda \leq 2.4$; this quantity is proportional to the area under $\alpha^{2} F$ and corresponds to a zone integral of $\gamma_{Q \nu} / \omega_{Q \nu}$. They were not including strong coupling corrections to $\mathrm{T}_{c},[15]$ however, which were unknown at the time of the original work. The strong-coupling limit[15] gives $T_{c} \propto \sqrt{\lambda \omega_{2}^{2}}$; this product $\lambda \omega_{2}^{2}$ corresponds to a zone integral of $\gamma_{Q \nu}$. We find that, for the fine mesh compared to the coarse (see the Table): $\lambda$ decreases by $70 \%$; $\mathrm{T}_{c}$ drops by $35 \%$ (at $\mu^{*}=0.13$ ); the Carbotte product decreases by $30 \%$; the strong-coupling limit factor drops by only $5 \%$. Hence the Carbotte ratio follows the change in $\mathrm{T}_{c}$ best, of these choices.

The behavior of $\lambda_{Q \nu} \propto \gamma_{Q \nu} / \omega_{Q \nu}^{2}$ does not faithfully represent the relative importance of modes for $\mathrm{T}_{c}$ except in the weak-coupling limit. In the strong coupling limit (which we do not claim is the case for $\mathrm{Li}$ ) it is $\gamma_{Q \nu}$ that gives the better picture. For another perspective we display in Fig. 6 an isosurface of $\gamma_{Q \nu}$ for the $\mathcal{L}$ modes. As expected from Fig. 3, it is dominated by contributions near the center of the (100) line. The isocontours form a blunt jack-like shape although the true symmetry is only four-fold. Our corresponding plots for $\lambda_{Q \nu}$ are almost like those of Profeta et $a l .[7]$ except that we seem to find somewhat larger contributions near the zone center.

In this paper we have clarified and supported our earlier suggestion[8] that denser zone sampling would be necessary to obtain accurate numerical results. We find in addition that careful sampling of the fine structure in the coupling is required even for obtaining a correct understanding of the microscopic origin of the pressure-induced increase in $\mathrm{T}_{c}$. In particular, we call into question the view (easily surmised from the earlier work $[7,8]$ even if not so claimed) that the impressive value of $\mathrm{T}_{c}$ in $\mathrm{Li}$ under pressure arises either from soft phonons near $\mathrm{K}$, or from a box-like set of regions in momenta along $(\eta, \eta, \zeta)$ with $\eta \sim 0.7$. These questions, and the strong polarization dependence of matrix elements, will be addressed in further work.

\section{Acknowledgments}

We acknowledge important advice from S. Y. Savrasov on the linear response code, and interactions with A. Lazicki, R. T. Scalettar, and C. S. Yoo. We thank U. Nitzsche for assistance in the use of the IFW cluster to perform the calculations. This work was supported by National Science Foundation grant Nos. DMR-0421810 and DMR-0312261. J.K. was supported by DOE grant FG02-04ER46111, and H.R. was supported by DFG (Emmy-Noether-Program).

\section{References}

[1] K. Shimizu et al., Nature 419, 597 (2002).

[2] S. Deemyad and J. S. Schilling, Phys. Rev. Lett. 91, 167001 (2003).

[3] V. V. Struzhkin et al., Science 298, 1213 (2002).

[4] J. J. Hamlin, V. G. Tissen, and J. S. Schilling, Phys. Rev. B 73, 094522 (2006).

[5] J. Sarrao et al., Nature 420, 297 (2002).

6] K. M. Lang et al., J. Low Temp. Phys. 114, 445 (1999).

[7] G. Profeta et al., Phys. Rev. Lett. 96, 047000 (2006).

[8] D. Kasinathan et al., Phys. Rev. Lett. 96, 047004 (2006).

[9] P. B. Allen, Phys. Rev. B 6, 2577 (1972); P. B. Allen and M. L. Cohen, Phys. Rev. Lett. 29, 1593 (1972).

[10] S. Y. Savrasov, Phys. Rev. B 54, 16470 (1996); S. Y. Savrasov and D. Y. Savrasov, ibid. 54, 16487 (1996).

[11] S. H. Vosko, K. Wilk and N. Nusair, Can. J. Phys. 58, 1200 (1980).

[12] J. S. Tse, Y. Ma, and H. M. Tutuncu, J. Phys.: Condens. Matt. 17, S911 (2005).

[13] L. Shi and D. A. Papaconstantopoulos, Phys. Rev. B 73, 184516 (2006).

[14] Z. P. Yin, S. Y. Savrasov, and W. E. Pickett, private communication.

[15] P. B. Allen and R. C. Dynes, Phys. Rev. B 12, 905 (1975).

[16] See J. P. Carbotte, Rev. Mod. Phys. 62, 1027 (1990) and references therein. 